\title{
Dynamic Model and Analysis of Asymmetric Telescopic Wing for Morphing Aircraft
}

\author{
Chen Lili ${ }^{\mathrm{a}}$, Guo Zheng, Wang Wenkai \\ Institute of Nearspace Technology, College of Aerospace Science and Engineering, National University of Defense \\ Technology, Changsha, China
}

\begin{abstract}
Morphing aircraft has been the research hot topics of new concept aircrafts in aerospace engineering. Telescopic wing is an important morphing technology for morphing aircraft. This paper describes the dynamic equations and kinematic equations based on theorem of momentum and theorem of moment of momentum, which are available for all morphing aircrafts. Meanwhile,as simplified, dynamic equations for rectangular telescopic wing are presented. In order to avoid the complexity using aileron to generate rolling moment, an new idea that asymmetry of wings can generate roll moment is introduced. Finally, roll performance comparison of asymmetric wing and aileron deflection shows that asymmetric telescopic wing can provide the required roll control moment as aileron, and in some cases, telescopic wing has the superior roll performance.
\end{abstract}

\section{Introduction}

Morphing aircraft has been investigated extensively because of its superior flight performance[1, 2]. Morphing aircraft can adapt various complicated flight regimes. The Defense Advanced Research Projects Agency (DARPA) developed Morphing Aircraft Program in 1998. At the beginning of 21 century, NASA showed its morphing aicraft project. Ordinarily morphing aircraft can be classified three types:micro morphing, small morphing and large morphing. Micro morphing includes variable camber[3, 4] and twist[5], small morphing contains variable tip and variable horizontal tail, while variable sweep[6,7],variable span[8] and folding wing belong to large morphing. This paper mainly concentrates on telescopic wing which is one of large morphing techniques.

Telescopic Wing is intended to change its wing span for various flight conditions to reduce drag. As aresult of increasing the wing span, the aspect ratio and wing area increase and the spanwise lift distribution decreases for the same lift[8].Telescopic wing has a long research history, many patents of invention have been declared[9], as shown in Fig.1, which is three patents about telescopic wing.

In this work, the dynamic and kinematic model are described to investigative performance of morphing aircraft. But, because the dynamic model involves some variable parameters with time, which leads to complexity of morphing aircraft. In order to obtain some basic characteristics, some hypotheses are put forward. Telescopic wing aircraft can change wing span and area during flight to realize better aerodynamic and control performance. Although much work has been done, technique of telescopic wing is not well developed.Some fundamental reseach work of telescopic wing was done focusing on dynamic model and analysis in this paper.

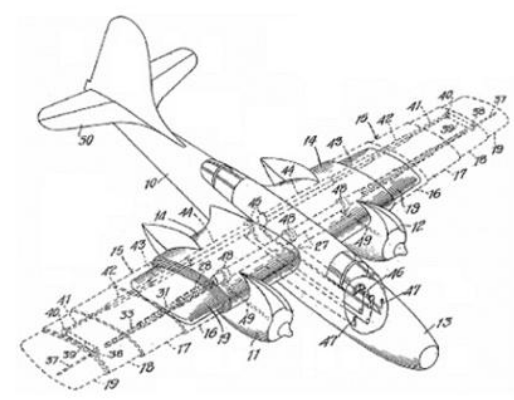

(a) Patent of variable erea by Gibson

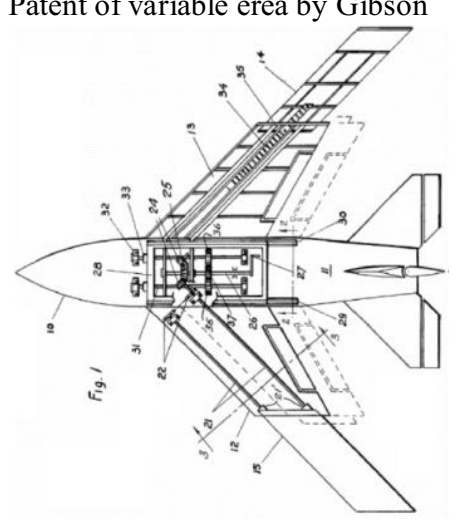

(b) Patent of telescopic wing by Kppenkin

\footnotetext{
a Chen Lili: doublely@foxmail.com
} 


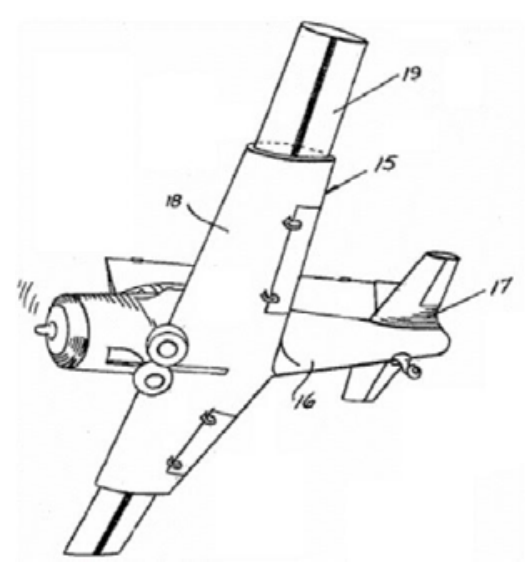

(c) Patent of variable erea by Murray

Figure.1 Patents of telescopic wings

\section{Morphing aircraft model}

\subsection{Dynamic model}

In this section the dynamic model of morphing aircraft is presented based on theorem of momentum and theorem of moment of momentum and also refers the symmetric wing dynamic model of ideal rigid-body, which is related to telescopic wing by Jin Dongping[10]. On the assumption that origin is located on symmetry position of fuselage, aircraft axes (or named as body axes)O-XYZ(as shown in Fig. 2) can be defined.In addition, inertial reference axes is defined as $\mathrm{O}_{\mathrm{A}}-\mathrm{X}_{\mathrm{A}} \mathrm{Y}_{\mathrm{A}} \mathrm{Z}_{\mathrm{A}}$ (as shown in Fig. 2) attached to ground (earch whirling ignored). The velocity and angular rate of morphing aircraft are $V_{\mathrm{A}}$ and $\omega_{A}$ relative to inertial reference axes,respectively. Usually, $V_{\mathrm{A}}$ and $\omega_{A}$ are projected on aircraft axes , marked as $V=\left(V_{x}, V_{y}, V_{z}\right), \omega=\left(\omega_{x}, \omega_{y}, \omega_{z}\right)$.

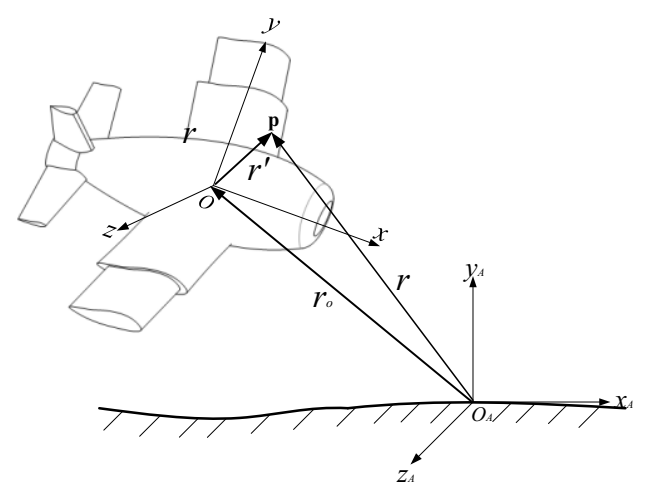

Figure 2 Chematic diagram of dynamic model

For any $\boldsymbol{d} \boldsymbol{m}$ of body, its position vetor is $\boldsymbol{r}_{\boldsymbol{i}}$ to inertial axes, the integral result can be expressed as:

$$
p=\int_{\Omega} r_{i} d m=\int_{\Omega}\left(r_{o}+r^{\prime}\right) d m
$$

According to theorem of moment of mass, the latter part can be noted as:

$$
S=\int_{\Omega} r^{\prime} d m
$$

Derivative expression is

$$
S=\dot{\circ}_{o}+\omega \times S_{o}
$$

Where $S$ and $\dot{S}_{o}$ are the derivative of instantaneous mass moment in inertial reference axes and aircraft axes, respectively. "。"represents the derivative to time in the aircraft axes.

Duo to $r_{o}=V$, thus

$$
p=m V+\stackrel{\circ}{S_{o}}+\omega \times S_{o}
$$

Where $m$ is mass of aicraft (including fuselage and wings ,also other parts),the moment of momentum relative to origin of aircraft axes can be expressed:

$$
\begin{aligned}
& L_{o}=\int_{\Omega} r_{i}^{\prime} \times v_{i} d m=\int_{\Omega} r_{i}^{\prime} \times\left(V+r_{i}^{\prime}\right) d m \\
& =S_{O} \times V+\int_{\Omega} r_{i}^{\prime} \times r_{i}^{\prime} d m
\end{aligned}
$$

Because aircaft axes has the rotation rate $\omega$ itself ,so

$$
L_{o}=S_{o} \times V+\int_{\Omega} r_{i}^{\prime} \times\left(\dot{r}_{i}^{\prime}+\omega \times r_{i}^{\prime}\right) d m
$$

In the light of definition,

$$
\begin{aligned}
& \int_{\Omega} r_{i}^{\prime} \times\left(\omega \times r_{i}^{\prime}\right) d m=\int_{\Omega}\left[\omega\left(r_{i}^{\prime} r_{i}^{\prime}\right)\right. \\
& \left.-r_{i}^{\prime}\left(\omega r_{i}^{\prime}\right)\right] d m=I \cdot \omega
\end{aligned}
$$

Where $I$ is the aircraft instantaneous inertia tensor relative to origin $\mathrm{O}$,the expression is as follows:

$$
\begin{aligned}
I & =\left[\begin{array}{ccc}
I_{x x} & -I_{x y} & -I_{x z} \\
-I_{y x} & I_{y y} & -I_{y z} \\
-I_{z x} & -I_{z y} & I_{z z}
\end{array}\right] \\
\text { So } \quad L_{o} & =S_{o} \times V+I \cdot \omega+\int_{\Omega}\left(r_{i}^{\prime} \times \dot{r}_{i}^{\prime}\right) d m
\end{aligned}
$$

$$
\text { Theorem of momentum: } \quad p=F
$$

To make it simple, the relative theorem of moment of momentum of any point can be demonstrated in the translation axes as in Fig.3:

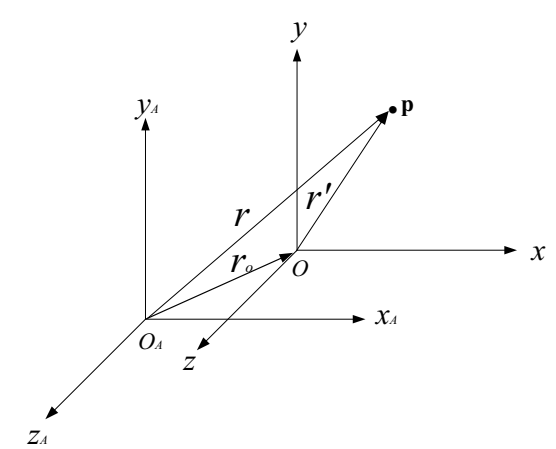

Figure 3 Inertial axes and translation axes

$$
L_{O i}=r_{i}^{\prime} \times m_{i} v_{i}
$$

Eq.(11) can be derivated, applying Newton second law to the result:

$$
\frac{d L_{O i}}{d t}=\frac{d r_{i}^{\prime}}{d t} \times m_{i} v_{i}+r_{i}^{\prime} \times m_{i} \frac{d v_{i}}{d t}=\frac{d r_{i}^{\prime}}{d t} \times m_{i} v_{i}+r_{i}^{\prime} \times F_{i}
$$


Due to

$$
\begin{gathered}
\frac{d r_{i}^{\prime}}{d t}=\frac{d r_{i}}{d t}-\frac{d r_{o}}{d t}=v_{i}-v_{o} \\
r_{i}^{\prime} \times F_{i}=M_{o i}
\end{gathered}
$$

Substituting Eq.(13) and Eq.(14) into Eq.(12) and integral yields the theorem of moment of momentum of whole aircraft

$$
\frac{d L_{o}}{d t}=\int_{\Omega} v_{i} d m \times V+M_{o}=p \times V+M_{o}
$$

Substituting Eq.(4) and Eq.(9) into Eq.(15) and considering vetor cross product relationship:

$$
\omega \times\left(S_{o} \times V\right)+V \times\left(\omega \times S_{o}\right)=S_{o} \times(\omega \times V)
$$

Taking an inertial derivative of the vector with respect to time in whirling reference frame[11], morphing aircraft flight dynamic model afer simplification under body axes yields

$$
\left\{\begin{array}{l}
m(V+\omega \times V)+\stackrel{\infty}{S}_{o}+\omega \times S_{o}+2 \omega \times \dot{S}_{o} \\
+\omega \times\left(\omega \times S_{o}\right)=F \\
I \cdot \omega+I \cdot \omega+\omega \times(I \cdot \omega)+S_{o} \times V+S_{o} \times(\omega \times V) \\
+\int_{\Omega}\left\{r_{i}^{\prime} \times \stackrel{\circ}{r}_{i}^{\prime}+\omega \times\left(r_{i}^{\prime} \times \stackrel{\circ}{r}_{i}^{\prime}\right)\right\} d m=M_{o}
\end{array}\right.
$$

Where $F$ is the force imposed on the aircraft under body axes, $M_{o}$ is the force moment relative to the origin of body axes caused by external force. Eq.(17) shows that motion parameters of telescopic wing aircraft are tightly related with the time variable of moving wing,for instance, $S_{o} 、 S_{O i} 、 I$ are functions of time. To rigid aircraft,wings maintain the constant relative position, then the equations reduce as:

$$
\left\{\begin{array}{l}
m(V+\omega \times V)+\omega \times S_{o}+\omega \times\left(\omega \times S_{o}\right)=F \\
I \cdot \omega+\omega \times(I \cdot \omega)+S_{o} \times V+S_{o} \times(\omega \times V)=M_{o}
\end{array}\right.
$$

If ,furthermore, center of mass is selected as the origin of body axes, Eq.(18) can be simplified as:

$$
\left\{\begin{array}{l}
m(V+\omega \times V)=F \\
I \cdot \omega+\omega \times(I \cdot \omega)=M_{o}
\end{array}\right.
$$

This equation can be used as center of mass motion equation and around center of mass motion equation in noninertial system.

Generally, moving wings have a small part mass of the whole aircraft and that product of inertia is little relatively.So, it is proper that $\boldsymbol{I}_{\mathrm{xy}}, \boldsymbol{I}_{\mathrm{yz}}, \boldsymbol{I}_{\mathrm{zx}}$ are equal to 0 , scalar quantity format of dynamic equations in the body axes can obtain in the following:

$$
\begin{aligned}
& m\left(V_{x}+\omega_{y} \cdot V_{z}-\omega_{z} \cdot V_{y}\right)+S_{o x}^{\infty 0}+\left(\omega_{y} \cdot S_{o z}-\omega_{z} \cdot S_{o y}\right) \\
& +2\left(\omega_{y} \cdot S_{o z}-\omega_{z} \cdot S_{o y}\right)+\omega_{y} \cdot\left(S_{o y} \omega_{x}-S_{o x} \omega_{y}\right) \\
& +\omega_{z} \cdot\left(S_{o z} \omega_{x}-S_{o x} \omega_{z}\right)=F_{x} \\
& m\left(V_{y}+\omega_{z} \cdot V_{x}-\omega_{x} \cdot V_{z}\right)+S_{o y}^{\infty}+\left(\omega_{z} \cdot S_{o_{x}}-\omega_{x} \cdot S_{o z}\right)(20) \\
& \left\{+2\left(\omega_{z} \cdot S_{o x}-\omega_{x} \cdot S_{o z}\right)+\omega_{z} \cdot\left(S_{o z} \omega_{y}-S_{o y} \omega_{z}\right)\right. \\
& -\omega_{x} \cdot\left(S_{o y} \omega_{x}-S_{o x} \omega_{y}\right)=F_{y} \\
& m\left(V_{z}+\omega_{x} \cdot V_{y}-\omega_{y} \cdot V_{x}\right)+S_{o z}^{\infty}+\left(\omega_{x} \cdot S_{o y}-\omega_{y} \cdot S_{O x}\right) \\
& +2\left(\omega_{x} \cdot S_{o y}-\omega_{y} \cdot S_{o x}\right)-\omega_{x} \cdot\left(S_{o z} \omega_{x}-S_{o x} \omega_{z}\right) \\
& -\omega_{y} \cdot\left(S_{o z} \omega_{y}-S_{o y} \omega_{z}\right)=F_{z} \\
& \left\{\begin{array}{l}
I_{x} \cdot \omega_{x}+I_{x} \cdot \omega_{x}+\left(\omega_{y}\left(I_{z} \cdot \omega_{z}\right)-\omega_{z}\left(I_{y} \cdot \omega_{y}\right)\right)+\left(S_{o y} V_{z}\right. \\
\left.-S_{o z} V_{y}\right)-S_{o y}\left(V_{x} \cdot \omega_{y}-V_{y} \cdot \omega_{x}\right)-S_{o z}\left(V_{x} \cdot \omega_{z}-V_{z} \cdot \omega_{x}\right) \\
+\int\left\{r_{i y}^{\prime} \cdot r_{i z}^{\infty}-r_{i z}^{\prime} \cdot r_{i y}^{\prime}{ }^{\prime}+\omega_{y}\left(r_{i y}^{\prime} \cdot r_{i x}^{\prime}-r_{i x}^{\prime} \cdot r_{i y}^{\prime}\right)\right.
\end{array}\right. \\
& \left.+\omega_{z}\left(\dot{r}_{i z}{ }^{\prime} \cdot r_{i x}{ }^{\prime}{ }^{\prime}-r_{i x}^{\prime} \cdot r_{i z}{ }^{\prime}\right)\right\} d m=M_{o x} \\
& I_{y} \cdot \omega_{y}+I_{y} \cdot \omega_{y}+\left(\omega_{z}\left(I_{x} \cdot \omega_{x}\right)-\omega_{x}\left(I_{z} \cdot \omega_{z}\right)\right)+\left(S_{o z} V_{x}\right. \\
& \left.-S_{O_{x}} V_{z}\right)+S_{O_{x}}\left(V_{x} \cdot \omega_{y}-V_{y} \cdot \omega_{x}\right)-S_{o_{z}}\left(V_{y} \cdot \omega_{z}-V_{z} \cdot \omega_{y}\right) \\
& +\int_{\Omega}\left\{r_{i z}{ }^{\prime} \cdot r_{i x}{ }^{\prime}-r_{i x}{ }^{\prime} \cdot r_{i z}^{\circ}+\omega_{z}\left(r_{i z}^{\prime} \cdot r_{i y}{ }^{\prime}{ }^{\prime} r_{i y}^{\prime}{ }^{\prime} \cdot r_{i z}{ }^{\prime}\right)\right. \\
& \left.-\omega_{x}\left(r_{i y}{ }^{\prime} \cdot r_{i x}{ }^{\prime}-r_{i x}{ }^{\prime} \cdot r_{i y}{ }^{\prime}\right)\right\} d m=M_{o y} \\
& I_{z} \cdot \omega_{z}+I_{z} \cdot \omega_{z}+\left(\omega_{x}\left(I_{y} \cdot \omega_{y}\right)-\omega_{y}\left(I_{x} \cdot \omega_{x}\right)\right)+\left(S_{o_{x}} V_{y}\right. \\
& \left.-S_{o_{y}} V_{x}\right)+S_{o_{x}}\left(V_{x} \cdot \omega_{z}-V_{z} \cdot \omega_{x}\right)+S_{o_{y}}\left(V_{y} \cdot \omega_{z}-V_{z} \cdot \omega_{y}\right) \\
& +\int_{\Omega}\left\{r_{i x}{ }^{\prime} \cdot r_{i y}{ }^{\prime}{ }^{\prime}-r_{i y}{ }^{\prime} \cdot r_{i x}{ }^{\prime}-\omega_{x}\left(r_{i z}{ }^{\prime} \cdot r_{i x}{ }^{\prime}-r_{i x}{ }^{\prime} \cdot r_{i z}{ }^{\prime}\right)\right. \\
& \left.-\omega_{y}\left(r_{i z}{ }^{\prime} \cdot r_{i y}{ }^{\prime}-r_{i y}{ }^{\prime} \cdot r_{i z}{ }^{\prime}\right)\right\} d m=M_{o z}
\end{aligned}
$$

Obviously,the dynamic equation series is very complex and has 6-DOFs that couple with each other.It is difficult to solve. Whereas, the equation is available for all morphing aircrafts and can be applied to kinds of ridid or flexible morphing aircraft ,in the previous assumption.

\subsection{Kinematic model}

In order to obtain the movement trajectory of morphing aircraft in inertial axes,it is essential to bulid the relatinship of inertial axes and body axes.As usual,the relationship of the two axes is denoted by three Euler angles. Their definitions[12] (Fig.4) as follws:

Angle of pitch $\varphi$ :the angle between the longitudinal axis of a flight vehicle $O x$ and the horizontal surface $x_{A} O z_{A}$.

Angle of yaw $\psi$ :the angle between the axis of $o x_{A}$ and the longitudinal axis $o x$ projection on the horizontal surface $x_{A} O z_{A}$.

Angle of roll $\gamma$ :the angle between the axis of $o y$ 
and vertical surface including the longitudinal axis $o x$ of the fight vehicle.

Coordinate transformations [12] between inertial axes and body axes are shown as

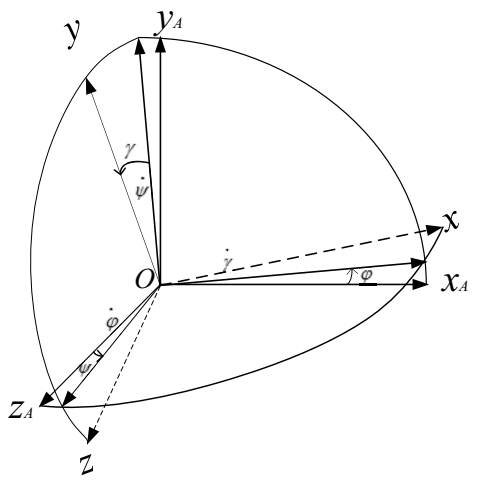

Figure 4 Relationship of Euler angles

$$
\begin{aligned}
& B_{A}=\left[\begin{array}{ll}
\cos \varphi \cos \psi \\
\cos \varphi \sin \psi \sin \gamma-\sin \varphi \cos \gamma \\
\cos \varphi \sin \psi \cos \gamma+\sin \varphi \sin \gamma \\
\sin \varphi \cos \psi & -\sin \psi \\
\sin \varphi \sin \psi \sin \gamma+\cos \varphi \cos \gamma & \cos \psi \sin \gamma \\
\sin \varphi \sin \psi \cos \gamma-\cos \varphi \sin \gamma & \cos \psi \cos \gamma
\end{array}\right]
\end{aligned}
$$

Then Kinematic equation in the inertial axes can be given by

$$
\left[V_{A x}, V_{A y}, V_{A z}\right]^{\mathrm{T}}=A_{B}\left[V_{x}, V_{y}, V_{z}\right]^{\mathrm{T}}
$$

Where $A_{B}=B_{A}^{T}$, the corresopnding scalar format is

$$
\left\{\begin{array}{l}
\frac{d x_{A}}{d t}=\cos \varphi \cos \psi V_{x}+(\cos \varphi \sin \psi \sin \gamma-\sin \varphi \cos \gamma) V_{y} \\
+(\cos \varphi \sin \psi \cos \gamma+\sin \varphi \sin \gamma) V_{z} \\
\frac{d y_{A}}{d t}=\sin \varphi \cos \psi V_{x}+(\sin \varphi \sin \psi \sin \gamma+\cos \varphi \cos \gamma) V_{y} \\
+(\sin \varphi \sin \psi \cos \gamma-\cos \varphi \sin \gamma) V_{z} \\
\frac{d z_{A}}{d t}=-\sin \psi V_{x}+(\cos \psi \sin \gamma) V_{y}+(\cos \psi \cos \gamma) V_{z}
\end{array}\right.
$$

The attitude of aircraft is described by three Euler angles $\varphi 、 \psi$ and $\gamma$. The anglar equations of motion can be obtained throught finding the transformation relation of Euler angles[13].First, $\varphi$ is formed by the angular rate $\varphi$ around $z_{A}$ axis, $\psi$ is from the angular rate of new axis $y^{\prime}$, which is projected on body axes as $[0, \psi \cos \gamma,-\psi \sin \gamma], \gamma$ is the rotation angle around axis $x$ in ${ }_{\gamma}$.Thus

$$
\left[\begin{array}{l}
\omega_{x} \\
\omega_{y} \\
\omega_{z}
\end{array}\right]=B_{A}\left[\begin{array}{l}
0 \\
0 \\
\varphi
\end{array}\right]+\left[\begin{array}{l}
0 \\
\psi \cos \gamma \\
-\psi \sin \gamma
\end{array}\right]+\left[\begin{array}{l}
\gamma \\
0 \\
0
\end{array}\right]
$$

$$
\left\{\begin{array}{l}
\omega_{x}=-\varphi \sin \psi+\gamma \\
\omega_{y}=\varphi \cos \psi \sin \gamma+\psi \cos \gamma \\
\omega_{z}=\varphi \cos \psi \cos \gamma-\psi \sin \gamma
\end{array}\right.
$$

Describing Eq.(24) in another form

$$
\left\{\begin{array}{l}
\frac{d \varphi}{d t}=\frac{1}{\cos \psi}\left(\omega_{y} \sin \gamma+\omega_{z} \cos \gamma\right) \\
\frac{d \psi}{d t}=\omega_{y} \cos \gamma-\omega_{z} \sin \gamma \\
\frac{d \gamma}{d t}=\omega_{x}+\tan \psi\left(\omega_{y} \sin \gamma+\omega_{z} \cos \gamma\right)
\end{array}\right.
$$

Eq.(25) is the angular equations of motion.

\section{Analysis for an example}

To make analysis simple, some hypothesis is necessary to define ahead,for example:

a) The aircraft is composed of three components: fuselage、 left moving wing and right moving wing.

b) All wings have no aileron.

c) Fuselage(including fixed wing part) maintains relative fixed position.

d) All wings have no dihedral. value.

e) Stretching rate of telescopic wing is some contant

f) Mass of left and right wing is $\boldsymbol{m}_{\boldsymbol{1}}$ and $\boldsymbol{m}_{\mathbf{2}}$, supposing that $\boldsymbol{m}_{1}=\boldsymbol{m}_{2}$.

Based on the above hypothesis, a relative telescopic wing dynamic model would be established. While the wings are in the retractile status, there is one point $\boldsymbol{O}$ locating on symmetry surface of fuselage, where

$$
S_{o x}=\int_{\Omega} r_{i x}^{\prime} d m=0, S_{o y}=\int_{\Omega} r_{i y}^{\prime} d m=0
$$

$\Omega$ represents the integral of whole aicraft.As is shown in Fig.5,stretching rates of left and right wing are $\boldsymbol{v}_{1}, \boldsymbol{v}_{2}$, respectively. Sweep angle is $\chi$. At the moment, only $S_{o y}=\int_{\Omega} r_{i y}^{\prime} d m=0$

Then,

$$
S_{o x}=m_{1}\left(d_{2}-d_{1}\right) \sin (-\chi), S_{o z}=m_{1}\left(d_{2}-d_{1}\right) \cos \chi(26)
$$

Where $d_{1}=v_{1} t, d_{2}=v_{2} t$.

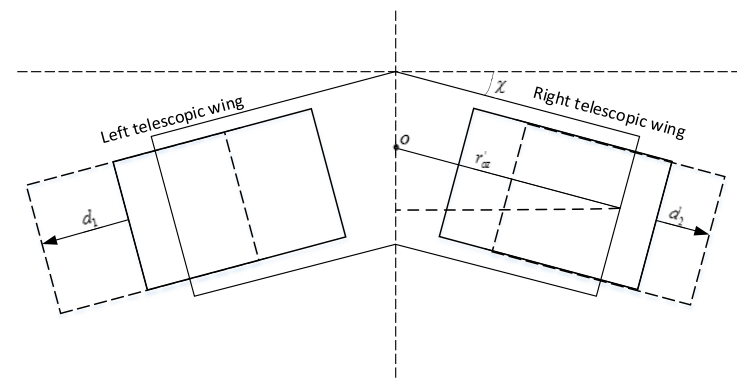

Figure 5 Sweep telescopic wing

The corresopnding scalar format is 


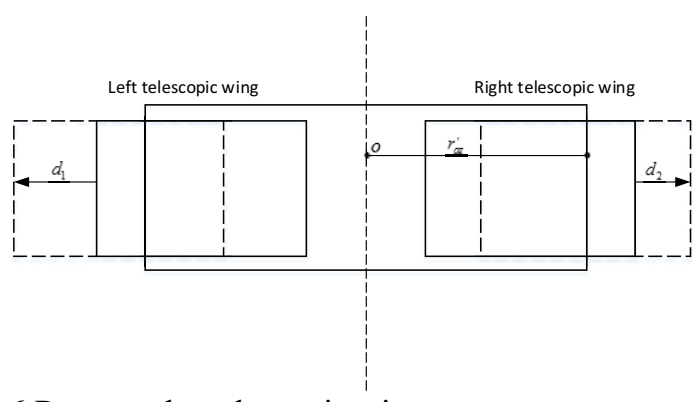

Figure 6 Rectangular telescopic wing

Futhrmore, accoring to the feature of rectangular telescopic wing, Eq.(26) can be expressed as

$$
S_{o x}=0, S_{o z}=m_{1}\left(d_{2}-d_{1}\right)=m_{1}\left(v_{2}-v_{1}\right) t
$$

Dynamic and kinematic equations are simplified as

$$
\begin{aligned}
& \left\{\begin{array}{l}
m\left(V_{x}+\omega_{y} \cdot V_{z}-\omega_{z} \cdot V_{y}\right)+\omega_{y} \cdot S_{o z}+2 \omega_{y} \cdot S_{o z} \\
+\omega_{x} \omega_{z} S_{o z}=F_{x} \\
m\left(V_{y}+\omega_{z} \cdot V_{x}-\omega_{x} \cdot V_{z}\right)+\omega_{x} \cdot S_{o z}+2 \omega_{x} \cdot S_{o z} \\
+\omega_{y} \omega_{z} S_{o z}=F_{y} \\
m\left(V_{z}+\omega_{x} \cdot V_{y}-\omega_{y} \cdot V_{x}\right)-\omega_{x}^{2} S_{o z}-\omega_{y}^{2} S_{o z} \\
=F_{z}
\end{array}\right. \\
& \left\{\begin{array}{l}
I_{x} \cdot \omega_{x}+I_{x} \cdot \omega_{x}+\left(\omega_{y}\left(I_{z} \cdot \omega_{z}\right)-\omega_{z}\left(I_{y} \cdot \omega_{y}\right)\right) \\
+S_{o z} V_{y}-S_{o z}\left(V_{x} \cdot \omega_{z}-V_{z} \cdot \omega_{x}\right)=M_{o x} \\
I_{y} \cdot \omega_{y}+I_{y} \cdot \omega_{y}+\left(\omega_{z}\left(I_{x} \cdot \omega_{x}\right)-\omega_{x}\left(I_{z} \cdot \omega_{z}\right)\right) \\
+S_{o z} V_{x}-S_{o z}\left(V_{y} \cdot \omega_{z}-V_{z} \cdot \omega_{y}\right)=M_{o y} \\
I_{z} \cdot \omega_{z}+I_{z} \cdot \omega_{z}+\left(\omega_{x}\left(I_{y} \cdot \omega_{y}\right)-\omega_{y}\left(I_{x} \cdot \omega_{x}\right)\right) \\
=M_{o z}
\end{array}\right.
\end{aligned}
$$

\section{Estimation of aerodynamic force}

The theoretical value of thin airfoil lift line slope is $2 \pi$, the value of thick wing increases with relative thickness of airfoil increasing.Experiment value is about $0.9 \times 2 \pi$, the correction value can approximately be expressed as

$$
C_{l}^{\alpha}=1.8 \pi(1+0.8 c)
$$

Where $c$ is thickness of airfoil .Because of tip effect, the slope of wing lift line is lower than airfoil ,

$$
C_{L}^{\alpha}=\frac{C_{l}^{\alpha}}{1+C_{l}^{\alpha} /(\pi \lambda e)}
$$

Where $C_{L}^{\alpha}, C_{l}^{\alpha}$ is the slope of wing and airfoil lift line, respectively, $\lambda$ is aspect ratio, $e$ is Oswald dfficiency factor, which is a function of aspect ratio and sweep angle. For rectangular wing,

$$
e=1.78\left(1-0.045 \lambda^{0.68}\right)-0.64
$$

Drag coefficient of wing can be estimated that[14]

$$
\begin{gathered}
C_{D}=C_{D 0}+C_{D i} \\
C_{D 0}=C_{f}\left(S_{W} / S\right), C_{D i}=K C_{L}^{2}
\end{gathered}
$$

Where $C_{f}$ is equivalent skin friction coefficient, $S_{w}$ is wetted area of wing, $S$ is reference area of wing, $K$ is damping factor by lift. At low velocity, equivalent skin friction coefficient of wing in turbulent flow can be approximately caculated as

$$
\begin{aligned}
& C_{f}=\frac{0.455}{(\operatorname{lgRe})^{2.58}} \\
& \text { Usually, } \quad K=\frac{1}{\pi \lambda e}
\end{aligned}
$$

For some specific rectangular telescopic wing, lift and drag force can be obtained from above formulae, then it is necessary to project them on inertial axes, the following is transformation matrix.

$$
B_{V}=\left[\begin{array}{ccc}
\cos \alpha \cos \beta & \sin \alpha & -\cos \alpha \sin \beta \\
-\sin \alpha \cos \beta & \cos \alpha & \sin \alpha \sin \beta \\
\sin \beta & 0 & \cos \beta
\end{array}\right]
$$

Where $\alpha$ (defined as angle of attack) is the angle between the axis $o x$ and velocity' projection on $x_{A} O z_{A}$ surface, $\beta$ (defined as angle of sideslip) is the angle between velocity vetor and $x_{A} o z_{A}$ surface.

\section{Analysis of roll performance}

\subsection{Rolling moment coefficient generated by aileron is caculated}

Increment of rolling moment coefficient by aileron deflection

$$
\mathrm{D}_{x}=\frac{\mathrm{D}_{x}}{q S b}=\frac{C_{l} q c y d y}{q S b}=\frac{C_{l} c y d y}{S b}
$$

Where $b$ is maximum wing span, $\mathrm{S}$ is maximum wing area, section lift coefficient of left wing can be given as

$$
C_{l}=C_{l}^{a} \frac{d a}{d d_{a}} d_{a}=C_{l}^{a} t_{a} d_{a}
$$

$\tau_{\mathrm{a}}$ is aileron dfficiency factor, which represents equivalent angles of attack of wing section with aileron when aileron deflects 1 degree, and can be estimated by $\tau_{a}=\left(c_{\mathrm{a}} / c\right)^{0.5}$.

Where $c_{\mathrm{a}}$ is chord of aileron.For rectangular wing, chord of wing $c$ is constant, consdering a couple of ailerons, through integral of $\Delta m_{x}$,

$$
m_{x}=\frac{C_{L}^{a} c t_{a}}{S b}\left(y_{2}^{2}-y_{1}^{2}\right) d_{a}
$$

Where $C_{L}^{a}$ and $t_{a}$ have been corrected by considering effect of practical flow, $y_{1}$ and $y_{2}$ is the starting and ending position of aileron along span .

\subsection{Rolling moment coefficient by asymmetric wing can be caculated}


Rolling moment coefficient:

$$
m_{x}=\frac{C_{L}^{a} a S_{a} \mathrm{D} L}{2 S b}
$$

Where $\Delta \mathrm{L}$ is difference value of left and right wing. $S \mathrm{a}$ is the actual wing area. Fixed wing is $2 \mathrm{~m}$, moving wing span is $1 \mathrm{~m}$, chord is $1 \mathrm{~m}$.If aileron is installed in the $25 \%$ of chord and its length takes up the whole moving wing span. NACA LS 0417 is selected as airfoil, then $C_{l}^{a}=2.0448 \pi, \mathrm{S}=6 \mathrm{~m}^{2}, \mathrm{~b}=6 \mathrm{~m}, \tau_{\mathrm{a}}=0.5$. The results of roll moment coefficient are shown in Fig.7 and Fig.8.

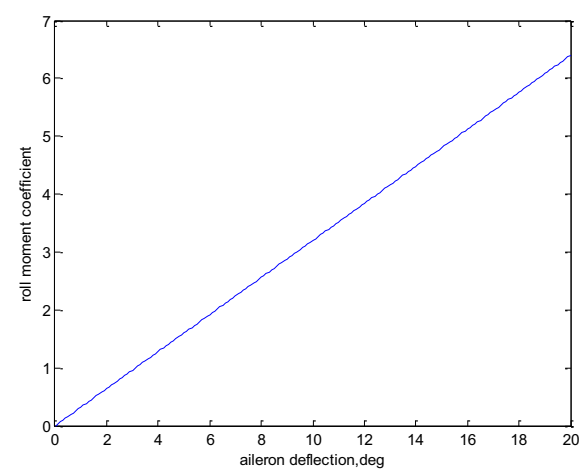

Figure 7 Rolling moment coefficient by aileron

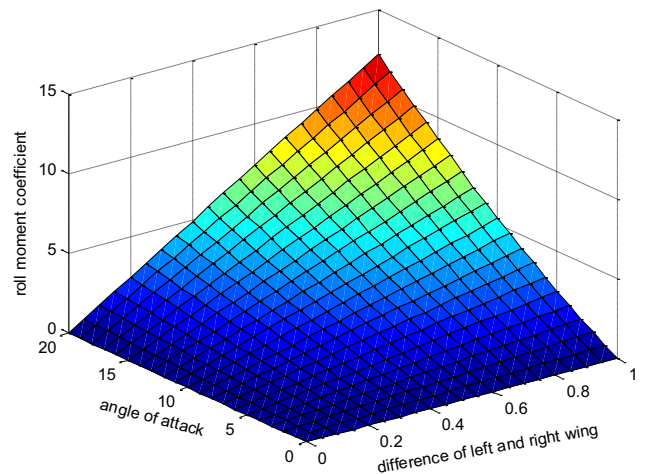

Figure 8 Rolling moment coefficient by telescopic wing

It can be seen from Fig.7 and Fig.8 that rolling moment coefficient generated by aileron is linear change, while that by elescopic wing is non-linear. When rolling moment coefficient of aircraft in a large angle of attack is easier controlled than in a small angle of attack.Meanwhile, rolling moment coefficient increases with $\Delta L$ increasing.

\section{Conclusions}

In this paper, the dynamic equations and kinematic equations of asymmetric telescopic wing aircraft are developed.From the equations, it is very complex and difficult to solve such equation series, because several parameters change with time.Hower, the model is versatile for any telescopic morphing aircraft, in some simple assumptions. This paper also bulid the model of rectangular telescopic wing with no sweep and dihedral.Some estimation formulae are applied to caculate the aerodynamic force. Results show that at low velocity high aspect ratio wing has better aerodynamic performance than low aspect ratio,while at high velocity low aspect ratio wing has superior performance. So, telescopic wing can enhance overall fight performance for a large velocity range.

The roll perfoamance also is evaluated by asymmetric wing span, aircraft can generate roll moment by change the stretching length of left and right wing. Results shows that roll moment coefficient varys evidently with difference value of telescopic wings' length and angle of attack. It is easy to controll the roll rate in this way. Moreover, roll performance of asymmetric wing is non-linear change. In the case of large angle of attack and $\Delta L$, telescopic wings behave better roll performance.

\section{References}

1. A., W. T. "Morphing Aircraft Systems : Historical Perspectives and Future Challenges," Journal of Aircraft Vol. 50, No. 2, pp. 337-350(2013)

2. Béguin, B., Breitsamter, C., Adams, N., et al. "Aerodynamic Investigations of a Morphing Membrane Wing," AIAA Journal Vol. 50, No. 11, pp. 2588-2599(2012)

3. Tomohiro, Y., Aya, S., and Yoshiyasu, H. "Development and Wind Tunnel Test of Variable Camber Morphing Wing," 22nd AIAA/ASME/AHS Adaptive Structures Conference. American Institute of Aeronautics and Astronautics, National Harbor, Maryland, (2014).

4. Vale, J., Leite, A., Lau, F., et al. "Aero-Structural Optimization and Performance Evaluation of a Morphing Wing with Variable Span and Camber," Journal of Intelligent Material Systems and Structures Vol. 22, No. 10, July 1, pp. 1057-1073,(2011)

5. Pecora, R., Amoroso, F., and Lecce, L. "Effectiveness of Wing Twist Morphing in Roll Control," Journal of Aircraft Vol. 49, No. 6, November-December, pp. 1666-1674,(2012)

6. Spearman, M. L., and Langley, N. "Aerodynamic Research at NACA / NASA Langley Related to the Use of Variable-Sweep Wings," 50th AIAA Aerospace Sciences Meeting including the New Horizons Forum and Aerospace Exposition. Nashville, Tennessee, (2012)

7. Hall, J. "Investigation of Variable Wing-Sweep for Applications in Micro Air Vehicles," Infotech@Aerospace. Arlington, Virginia, (2005)

8. Bae, J.-S., Seigler, T. M., and Inman, D. J. "Aerodynamic and Static Aeroelastic Characteristics of a Variable-Span Morphing Wing," Journal of Aircraft Vol. 42, No. 2, March-April, pp. 528-534,(2005)

9. Junfu, L., Junqiang, A., Shitu, L., et al. "Research on Telescopic Wing Morphing Technology," Advances in Aeronautical Science and Engineering Vol. 3, No. 1, pp. 2-5,2012 [10] Dongping, J., and Wenmei, Z. "Longitudinal flight dynamics of morphing aircraft with telescopic wings," Chinese Journal of Applied 
Mechanics Vol. Vol.31 No.3, pp. 468-472,(2014)

11. Adam, N., John, V., Leland, C., et al. "Morphing Aircaft Dynamical Model: Longitudinal Shape Changes," AIAA Atmospheric Flight Mechanics Conference and Exhibit. American Institute of Aeronautics and Astronautics, Honolulu, Hawaii, (2008)

12. Peiran, J., Kejun, C., and Li, H. Ballistics of Remote Rocket(In Chinese). China Changsha National University of Defense Technology Press, (1993)
13. Zhenping, F., Wanchun, C., and Shuguang, Z. Flight Dynamics of Aerial Vehicle(In Chinese). China Beijing: Beihang University Press, (2005)

14. Adam, N., John, V., Thomas, S., et al. "Six Degree-of-Freedom Dynamical Model of a Morphing Aircraft," AIAA Atmospheric Flight Mechanics Conference. American Institute of Aeronautics and Astronautics, Chicago, Illinois, (2009) 